\title{
A Cloud-based Driver Monitoring for Inefficient Driving Behavior using OBD2 Telematics
}

\author{
Swati Dixit \\ $\mathrm{BE}$ (Computerscience) \\ Modern Education Society's \\ College of Engineering \\ Pune-411001,India
}

\author{
Priya Thomas \\ $\mathrm{BE}$ (Computer Science) \\ Modern Education Society's \\ College of Engineering \\ Pune-411001,India
}

\author{
Chirag Agarwal \\ BE(Computer Science) \\ Modern Education Society's \\ College of Engineering \\ Pune-411001,India
}

\begin{abstract}
This paper proposes a cloud-based vehicular data acquirement and analytics system for real-time driver conduct monitoring, trip analysis, and vehicle diagnostics. It comprises of an OnBoard Diagnostics (OBD2) port to Bluetooth technology, a mobile application running on a smart phone, and a cloud based backend. The mobile app envisions both real-time data from sensors and alerts. A web based interface is rendered to gain the backend information. The cloud detects rash driving in real time based on the sensor data provided through the OBD port. Diachronic data is also used by the backend to detect driving anomalies and to foretell the hindering sensor failures.
\end{abstract}

\section{General Terms}

Internet of Things

\section{Keywords}

Driver Monitoring, Internet of Things, OBD2, Vehicle Diagnostics, Smart Phone.

\section{INTRODUCTION}

Many automobile drivers are aware of the driving behavior and habits that can result in incompetent and vulnerable driving. However, it is often the case that these same drivers inadvertently display these inefficient and dodgy driving behaviors in their everyday driving activity. This system intends to create an applicative and fugal way to capture / measure/evaluate incompetent, wasteful and unprotected driving with the technical details for evaluation of Real Time Car Supervising like Performance, Diagnostics (Using Diagnostics Trouble Code - DTC), Fuel intake \& Autonomy and Emanation from a Vehicle. The proposed solution consists of a mobile application, running on an Android Smartphone, paired with a compatible OBD2 (On-board diagnostics 2) reader.

\section{RELATED WORKS}

\subsection{Knowledge based real-time car monitoring and driving assistance}

Contemporary vehicles are outfitted with several Electronic Control Units (ECUs) coordinating and supervising internal components and subsystems, communicating over one or more car network buses. International regulations today mandate all new vehicles must support the OBD2 protocol and be equipped with an OBD-compliant interface to provide direct access to data in the internal automotive network. Furthermore, in case of malfunctions, DTC values are stored in the car and can be later retrieved by maintenance technicians using proper tools. This paper enhances framework in, able to interpret vehicle data extracted via OBD2, integrate environmental information and detect potential risk factors. Besides providing warnings for that, now the system gives suggestions during driving and evaluates car efficiency and environmental impact. It is fully compliant with widely available smart phones. By means of properly devised processing and fusion algorithms, the system is able to identify and classify given high-level events and conditions, based on low level data streams. The proposed framework has been implemented in a prototypical mobile software system, using the Apple iPhone1 Smartphone as reference platform. The experimental evaluation has been carried out in several real-world test drives under different conditions, evidencing both feasibility and usefulness of the approach.

\subsection{Smart phone based approach to monitor driving behavior and sharing of statistic}

In recent years, many researchers and industries are working on VANET and trying to implement the concepts in real world. Many VANET systems are proposed and tested on simulation. Vehicular ad hoc networks (VANETs) are being formulated to provide on demand wireless communication infrastructure among vehicles and authorities. Such an infrastructure is expected to deliver multiple road safety and driving assistance applications. Vehicles will be equipped with sensors and communication devices that will allow them to cooperate with each other. Vehicles can exchange different type of information as per requirements on demand for specified application. It aims on supporting and improving data collection and distribution, in this paper a smart phone based platform is designed that exploits low-cost dedicated hardware to interact with sensors on board and in the vehicle surroundings.

\subsection{Mobile application for monitoring inefficient and unsafe driving behavior}

Recently, in the mobile application market, several applications have emerged that pair the power of a mobile device with the information available through the use of an OBD2 reader. These applications tend to be directed toward auto enthusiasts, developing features that concentrate on measuring vehicle performance and troubleshoot mechanical issues. Other applications are surfacing that focus on environmental concerns. These applications concentrate on factors like measuring a driver's carbon footprint and fuel consumption. Some of these applications include features that can detect safety issue (e.g. issues with the vehicle's stability control system). However, these features are focused on detecting mechanical issues with safety equipment, not on identifying real time concerns with the driver's behavior or environment. 


\section{SOLUTION ARCHITECTURE}

The same basic principles that drove the first car engines are still used today. However, modern automotive engines have evolved to meet today's power, efficiency and emission standards. To achieve that, sensors are being used in each subsystem of the vehicle, which continuously monitor the states and are used as a feedback to control the inputs for the engine to gain the maximum in means of power, efficiency and emission standards. All the sensors are connected to the Power Train Control Module (PCM).

\subsection{Data gathering}

There is a special circuit integrated to the PCM, which is designed to monitor emission control systems and key engine components. It is called On-Board Diagnostics (OBD) which refers to a vehicle's self-diagnostic and reporting capability. Initially came the OBD1 and later OBD2 was introduced. OBD1 is connected to the dashboard console, so that the port can be diagnosed and data can be read. Whereas, OBD2 is remotely used to diagnose ports, and reads data through a Bluetooth connection. As such, it is easier to diagnose a problem remotely if you have a car made with anOBD2 system.OBD2 specifications only comprise the Physical Signal Layer (PSL) and OBD2 Data Communication Layer (DCL) of the ISO/OSI model. Though the system is able to retrieve all possible vehicle parameters via the OBD2interface, the analysis focused on: coolant temperature, engine load, throttle position, RPM (Revolutions per Minute), vehicle speed, MAF (Mass Air Flow), fuel level, upstream and downstream oxygen sensor.

\subsection{Server configuration}

\subsubsection{Configuration settings with cloud}

The proposed system records driving behavior of vehicles and uploads to the cloud server for further solicitations. The upload will include the data collected from the sensors during the unusual behavior of the driver as well as the location where the driver was at that particular instant. For an example, If the system detects the vehicle unsafe driving behavior, the vehicle location will be updated in clouds and an alert message will be issued to precise user. The analyses are performed on real time data as well on archived data that are collected over a long period of time. While some of these analyses are performed within the app, more complex and resource consuming ones are performed in the backend. The backend being talked about here is the cloud server used. Data are forwarded to the backend using HTTP messages.

\subsubsection{Cloud space purchase}

The proposed system is capable of collecting, storing, and analyzing vehicular data for a long period of time. The mobile app pulls the vehicular data using an OBD2 to Bluetooth interface. The collected data is then pre-processed at the Smartphone to detect interesting events. The preprocessed data is then sent to backend cloud servers using the Smartphone's 3G/4G connection. It will be using the cloud server name Everdata. Cloud space on Everdata will be purchased by registering through their official site for storing the pre-processed as well as post-processed information.

\subsubsection{Creation of nodes}

At present traffic safety has become one of the most important issues in vehicle technologies. Using the vehicle to vehicle communication model drivers can share the statistics of the vehicle in the range including the vehicle location, health, driving, etc. A Vehicular Ad Hoc Network or VANET is a technology that uses moving cars as nodes in a network to create a mobile network. VANET turns every participating car into a wireless router or node, allowing cars approximately 100 to300 m of each other to connect and, in turn, create anetwork with a wide range. As cars fall out of thesignal range and drop out of the network, other carscan join in, connecting vehicles to one another so thata mobile Internet is created.

\subsubsection{Node authentication}

Main benefit over using pre-implemented mobile technology as a communication media it gives all the security and wide area connectivity advantages to vehicle ad-hoc network. As every mobile phone having its registered and unique identity it can be utilized as identity of every vehicle node.

\subsection{Data normalization}

A user has to pay for each megabyte that is consumed over the $3 \mathrm{G} / 4 \mathrm{G}$ network. Therefore, the network bandwidth usage of the app is an important fact to consider. Hence, the CEP queries are used in the mobile app to filter out uninteresting events and only the useful data are transmitted over the 3G/4G network. Complex Event Processor (CEP) queries are also used to collect and send only the aggregated data for certain

types of sensor readings. Such reduction in data not only reduces the bandwidth requirement, but also minimizes power consumption of the Smartphone. 


\subsection{Mathematical techniques}

\subsubsection{K-Means clustering algorithm}

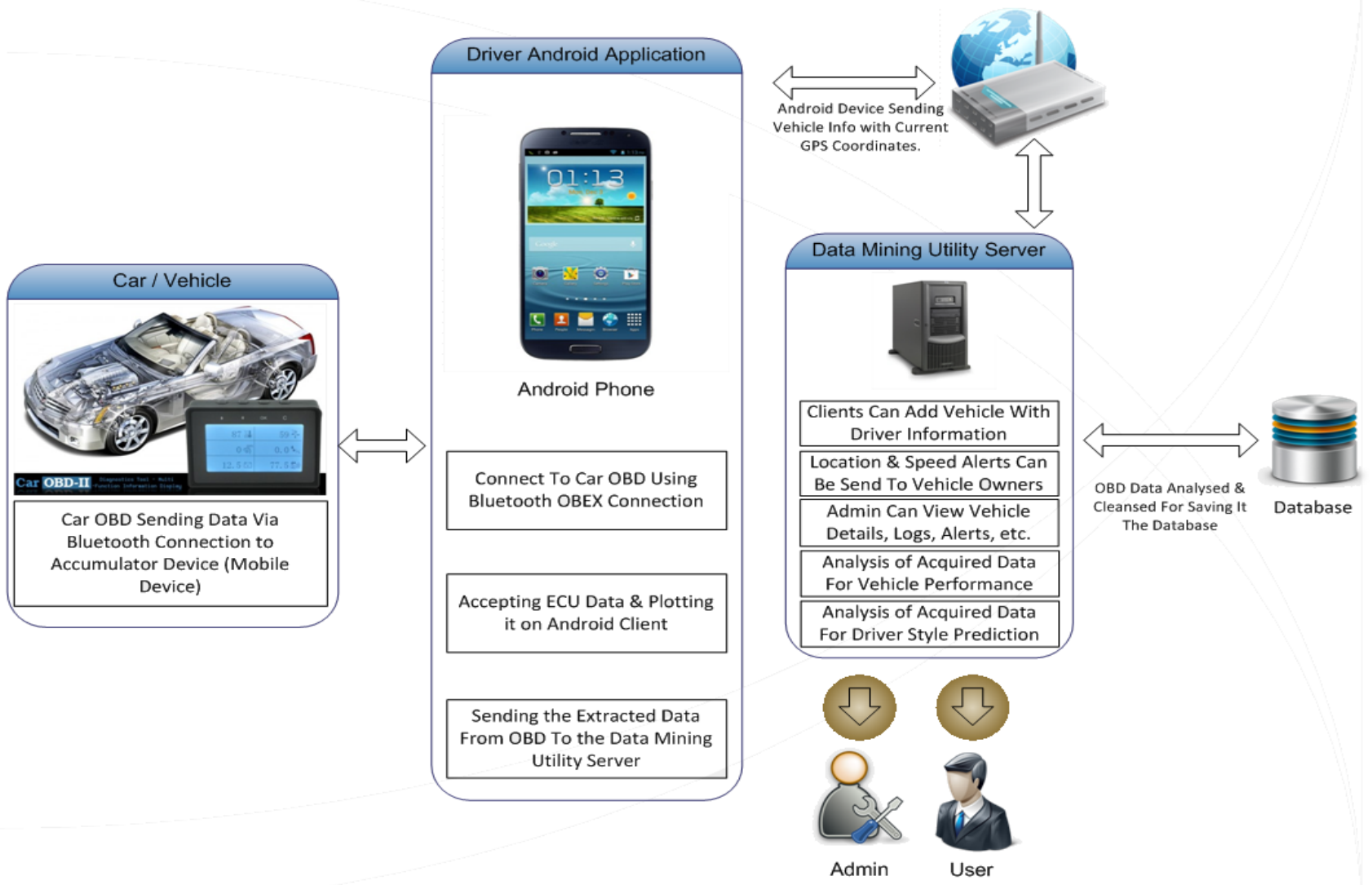

Fig 1:Architecture of the proposed system

$\mathrm{K}$-means clustering is a way of vector quantization, originated from signal processing, that is famous for cluster analysis in data mining. K-means clustering focuses to partition $n$ observations into $\mathrm{k}$ clusters in which each observation belongs to the cluster with the nearest mean, serving as a prototype of the cluster. This results in a partitioning of the data space into Voronoi cells.

\subsection{Data analysis}

Data analysis involves driver monitoring which includes two aspects:

\subsubsection{Reckless driving}

Reckless driving is a serious risk to the driver as well as general public. If a methodology to detect reckless driving can be developed, it will be fruitful for vehicle owners, general people, drivers, insurance providing companies, and many other stakeholders who would not want to put time and money at stake for the consequences of reckless driving reckless driving detection, which is a mobile phone based rash driving detection system. There, reckless driving behaviour was detected with the help of lateral and longitudinal acceleration. Many surveys demonstrated that the sudden change of the longitudinal acceleration is a good way to detect reckless driving. Hence, longitudinal acceleration to detect reckless driving was also used. The proposed solution for detecting reckless driving include following two steps:

\subsubsection{Pre-processing within application}

The speed of the vehicle can be obtained in real time from the OBD2 adapter. As it is the acceleration/deceleration which is of importance, the CEP engine converts the speed input into acceleration/deceleration input by taking consecutive speed readings. Calculated acceleration/deceleration is compared with an already defined threshold value to check if it is reckless or not. The threshold value of $4.5 \mathrm{~ms}-2$, as per the recommendation was used.

\subsubsection{Processing at backend}

At the backend, data gathered from the application are formatted by the WSO2 BAM as hourly, weekly, monthly, and three months' aggregates and collected in an SQL database ready for the web portal. And, when the aggregate for a given time span is above the already defined threshold value, notification in the form of alerts can be sent to both the drivers and other stakeholders

\subsubsection{Detection of driving anomalies}

Detecting vehicle driver patterns has become a principal research area due to the increasing concern about fuel consumption efficiency and safe driving, amount of being distributed on the accelerator and brake pedals. Adding on it was also found that it the acceleration pattern which is unique of each driver and not the speed pattern. A hidden Markov model was used to identify the difference between the present driving pattern and the past patterns of a (driver, vehicle) combined. Detection of driving anomalies happens in the following stages: 


\subsubsection{Preprocessing}

As in the use case of reckless driving detection, it is the acceleration that is of concern.CEP engine transforms the speed streams into acceleration streams. These acceleration streams are then converted to acceleration transition streams which consist of the timestamp, previous acceleration, and the current acceleration. These acceleration streams are transmitted to the backend. These streams are used by the CEP in real time for calculating whether the readings are in accordance with the normal driving pattern. The same stream is also used by BAM to update the model daily.

\subsubsection{Application of data analysis phase \\ 3.5.3.1 Receiving data from OBD2 layer}

The app is capable of connecting to the ELM-327 adaptor via Bluetooth and communicating with the vehicle using OBD2 Parameter IDs (PIDs). Each PID provides certain information about the vehicle, e.g., speed, rpm, fuel consumption, and error codes. The received data are logged inside the app and displayed in the user interface in real time. The architecture of the app is also extensible where the PIDs can be added dynamically.

\subsubsection{Monitoring vehicle}

The mobile app consists of a Complex event processor. CEP can be regarded as a service that receives and matches lower level events and generates higher-level events in real time. Simply, it is a component that responds to event streams in an event driven manner. Hence, CEP has the capability to detect relevant events in incoming data streams according to a predefined set of queries. For example, queries can be added to alert the driver, if the vehicle is running with a high rate of fuel consumption or high coolant temperature for a considerably long period of time. CEP queries can be also used to summarize trip details in real time, e.g., average fuel consumption for the ongoing trip.

\subsubsection{Selective transmission of backend data}

Complex event processing is also utilized at the backend. The $\mathrm{CEP}$ at the backend is responsible for detecting more complex driver and engine anomalies (e.g., reckless driving) and generating alerts in real time. Some of these alerts are pushed back (as push notifications) to the mobile app and notified to the driver. For example, warn the driver for reckless behavior and speed alters based on known GIS (Geographic Information System) information. The system is capable of performing long term analyses too. By identifying patterns, it should be able to predict undesirable outcomes such as potential failures of sensors. This is enabled by using a Business Activity Monitor as the long term analyzer at the backend. BAM is a solution primarily intended to provide a real-time summary of business activities and is capable of collecting, storing, and analyzing data. Binary values of 1 are assigned to the acceleration/deceleration values above the threshold and binary 0 is assigned for values below the threshold. The total number of 1's is then counted over a predefined time interval (e.g., 2 minutes). The counts are then classified according to the driving cycle (i.e., traffic, normal, and highway) of the trip. A driving cycle is a series of data points representing the speed of a vehicle versus time. Driving cycle is determined by the average speed of the vehicle throughout 10 minutes. The counts, together with the detected driving cycle are sent to the backend server periodically. Acceleration and deceleration values are sent as two separate streams.

\subsection{Evaluation}

Feature Extraction to get driving behavior of driver uses the following evaluation techniques:

\subsubsection{Fuel consumption and autonomy \\ 3.6.1.1 Injected fuel}

Injected Fuel $=$ MAF $/[($ fuel factor $) *($ fuel density) $]$ in lit/s

Where, fuel factor $=14.7 \%$,

fuel density $=720 \mathrm{~g} / \mathrm{l}$

3.6.1.2 Emission

Upstream Emission $=\mid$ Max - Min $\mid<5$

Downstream Emission $=|\operatorname{Max}-\operatorname{Min}|>5$

3.6.1.3 Speed

Speed Limit $=|\mathrm{Sc}-\mathrm{So}|>10$

where $\mathrm{Sc}=$ Current Speed,

So=Optimum Speed $=45 \mathrm{~km} / \mathrm{hr}$

3.6.1.4 Engine Coolant Temperature

Temp Rise $=\mid$ Ect - Ecot $\mid>3$

Where, $\mathrm{ECt}=$ Engine Coolant temperature,

ECot=Optimal Engine Coolant temperature $=104$ degree

Celsius

3.6.1.5 Fuel Consumption

Fuel Consumption =VSS / Injected fuel

\subsection{Roles}

3.7.1 Owner of the vehicle or the administrator Once a vehicle is bought, it is sold to many people at many times. Since all these users might not use the vehicle in a good way, it is best if a person could know the condition of a vehicle before purchasing. Currently available systems are only capable of detecting faulty conditions once the fault arises. Hence, a system which could perform a detailed analysis to predict such failures is much more beneficial for a buyer. Also, it is beneficial for the sellers from the sense that they could emphasize and prove the good condition of the vehicle they are planning to sell. Also, for the owner knowing the condition of the vehicle is important since it makes him feel comfortable if a failure is not to occur in the near future.

\subsection{Application}

\subsubsection{State agencies}

OBD plays an important role where vehicle inspection and maintenance programs are required.

\subsubsection{Vehicle owners}

OBD serves as an early warning system that alerts you to the potential need for vehicle repair through the" Check Engine" light on the dashboard of the vehicle.

\subsubsection{Vehicle and engine manufacturers}

OBD systems are required by EPA to be installed on light-duty vehicles and trucks, as well as heavy-duty engines.

\section{CONCLUSION}

The proposed solution proposes a practical and an economical way to capture, measure or evaluate inefficient, uneconomical and unsafe driving with the details about Performance, Diagnostics, Fuel Consumption and Autonomy and Emission from a Vehicle. It consists of a mobile application, running on 
an Android Smartphone, paired with a compatible OBD reader. There are several measurements, which are used alone in combination, that can help indicate if a driver is driving unsafe or inefficiently. Furthermore, the app visualizes realtime readings from vehicular sensors and notifications pushed from the cloud backend. Hence, various papers have been surveyed which would help the app to identify several components which will be required to develop the proposed system. Various algorithms have also been identified which may prove useful in the implementation of the system. If the majority of drivers can be alerted of dangerous behavior, situations and traffic conditions, there would consequently be fewer road accidents and fatalities. Such systems could also be useful to lower traffic jams by informing drivers of shortest routes to their destinations and also by informing relevant authorities to problems causing the jams. The ideation is that Smartphone-based vehicle will be a crucial part of the traffic system in the future, especially in developing countries.

\section{ACKNOWLEDGMENTS}

This research was extensively supported by the institution Modern Education Society's College of Engineering, Pune, India. We are thankful to Prof. Shalaka Deore who provided expertise that greatly assisted the research and improved the manuscript significantly.

\section{REFERENCES}

[1] Malintha Amarasinghe, Sasikala Kottegoda, Asiri Liyana Arachchi, Shashika Muramudalige, H. M. N. Dilum Bandara, Afkham Azeez: Cloud-Based Driver Monitoring and Vehicle Diagnostic with OBD2 Telematics. In: 2015 International Conference on Advances in ICT for Emerging Regions (ICTer)

[2] Ruta, M., Scioscia, F., Gramegna, F., Di Sciascio, E.: A Mobile Knowledge-Based System for On-Board Diagnostics and Car Driving Assistance. In: The Fourth International Conference on Mobile Ubiquitous Computing, Systems, Services and Technologies (UBICOMM2010), IARIA (2010) 91-96
[3] Colucci, S., Di Noia, T., Pinto, A., Ragone, A., Ruta, M., Tinelli, E.: A non-monotonic approach to semantic matchmaking and request refinement in emarket places. International Journal of Electronic Commerce 12(2) (2007) 127-154

[4] Ashutosh K,Piyush.K: Smart phone based approach to monitor driving behavior and sharing of statistic. In: 2014 Fourth International Conference on Communication Systems and Network Technologies

[5] Lin, C., Li, C.C., Yang, S.H., Lin, S.H., Lin, C.Y.: Development of On-Line Diagnostics and Real Time Early Warning System for Vehicles. In: Sensors for Industry Conference, 2005. (2005) $45-51$

[6] Lin, C.E., Shiao, Y.S., Li, C.C., Yang, S.H., Lin, S.H., Lin, C.Y.: Real-Time Remote Onboard Diagnostics Using Embedded GPRS Surveillance Technology. Vehicular Technology, IEEE Transactions on 56(3) (2007) 1108-1118

[7] Chen, Y., Xiang, Z., Jian, W., Jiang, W.: Design and implementation of multisource vehicular information monitoring system in real time. In: Automation and Logistics, 2009. ICAL '09. IEEE International Conference on. (August 2009) $1771-1775$

[8] Kargupta, H., Bhargava, R., Liu, K., Powers, M., Blair, P., Bushra, S., Dull, J., Sarkar, K., Klein, M., Vasa, M. Handy, D.: Vedas: A mobile and distributed data stream mining system for real-time vehicle monitoring. In Berry, M.W., Dayal, U., Kamath, C., Skillicorn, D.B., eds.: SDM, SIAM (2004)

[9] Choi, S., Kim, J., Kwak, D., Angkititrakul, P., Hansen, $\mathrm{J} .:$ Analysis and Classification of Driver Behavior using In-Vehicle CAN-Bus Information. In: Biennial Workshop on DSP for In-Vehicle and Mobile Systems. (June 2007) 